\title{
Diferencias en ansiedad escolar en alumnado ecuatoriano de bachillerato según sexo y curso
}

\author{
José Manuel García ${ }^{1}$, Carlos Édisson Jiménez ${ }^{2}$, Nancy Cargua ${ }^{2}$, y \\ María del Pilar Aparicio ${ }^{1}$ \\ ${ }^{1}$ Universidad de Alicante (España); ${ }^{2}$ Universidad Central del Ecuador (Ecuador)
}

En la actualidad, la ansiedad escolar es considerada por el ámbito científico como un trastorno emocional, que influye desde la infancia hasta la adolescencia. Los estudios previos que tratan este trastorno en población infanto-juvenil informan de una alta prevalencia a nivel mundial alrededor de entre el 10\% y el 20\% del alumnado. Sin embargo, en el país de Ecuador, esta área de investigación permanece desatendida. Por este motivo, el objetivo del presente estudio fue observar si existen diferencias en ansiedad escolar en función del sexo y curso para 720 adolescentes ecuatorianos entre 15 y 18 años de edad, matriculados entre $1^{\circ}$ y $3^{\circ}$ de bachillerato. Para ello, se utilizó el Inventario de Ansiedad Escolar en Secundaria (IAES). Los resultados arrojan puntuaciones significativamente más altas en el sexo femenino para el total del IAES, así como todos los factores de la escala. Asimismo, se observaron las principales diferencias estadísticamente significativas entre primero y tercero de bachillerato. En conclusión, estos resultados son similares a estudios previos realizados en otros países, lo que aboga por la importancia de diseñar y aplicar programas de intervención y prevención de la ansiedad escolar en el ámbito educativo.

Palabras clave: Ansiedad escolar, bachillerato, sexo, curso, problemas emocionales.

Differences in school anxiety in Ecuadorian high school students across gender and academic year. Currently, school anxiety is regarded by the scientific world as an emotional disorder, influencing from childhood to adolescence. Previous studies treating this disorder in infant-youth population report a high prevalence worldwide of about 10 to 20 per cent of pupils. However, in Ecuador, this area of research remains neglected. For this reason, the objective of this study was to see if there are differences in school anxiety according to sex and course for 720 Ecuadorian adoelscents between 15 and 18 years of age, enrolled between 1st and 3rd grade. For this purpose, the Inventario de Ansiedad Escolar en Secundaria (IAES) was used. The results show significantly higher scores in the female sex for the total of the IAES, as well as all the scale factors. Also, the main differences were observed statistically significative betewwn first and third of high school. In conclusion, these results are similar to previous studies carried out in other countries, which advocates the importance of designing and implementing programmes for intervention and prevention of school anxiety in the field of education.

Keywords: School anxiety, high school, sex, course, emotional problems.

Correspondencia José Manuel García Fernández. Universidad de Alicante, Facultad de Educación. Apdo. Correos, nº 99. C.P.: 03080. San Vicente del Raspeig, Alicante (España). E-mail: josemagf@ua.es 
El campo en el estudio de la psicología y la neurociencia en Ecuador han avanzado en los últimos años. No obstante, es mucho menor el estudio científico relacionado con las emociones y sus reacciones de carácter cognitivo, motor y psicofisiológico que un sujeto puede percibir ante diversos contextos escolares que se determinan como amenazas para el individuo (Jiménez-Ayala, 2017). Y ahí entra en juego la ansiedad escolar.

Pese a estimar la ansiedad como una de las problemáticas de carácter emocional más importantes a nivel mundial (Batxer, Scott, Vos, y Whiteford, 2013; Steel et al., 2014), en Ecuador se desconoce cómo influye la ansiedad escolar en edades adolescentes. A este respecto, se estima necesario el estudio de la misma, pues ya la abundante exigencia y las constantes transformaciones sociales, están provocando que una parte importante de la población declare sufrir o haber sufrido algún desorden vinculado con la ansiedad, tanto en población infanto-juvenil (Costello, Egger, y Angold, 2005; Ollendick, y Seligman, 2005), como en edades adultas (Kessler et al., 2007).

De hecho, muchas veces nos viene a la mente que este tipo de desórdenes son más concurrentes en edades adultas. Sin embargo, muchos trastornos ansiógenos se inician en la niñez siendo, por tanto, una de las dificultades anímicas que repercuten mayormente en el desarrollo de la población infantil (Beesdo, Knappe, y Pine, 2009), alargándose en el tiempo dependiendo de la gravedad del trastorno (Dadds et al., 1999).

En consecuencia, y pese a contemplar que la ansiedad y los procesos de miedo son situaciones frecuentes en la infancia (Méndez, Inglés, Hidalgo, García-Fernández, y Quiles, 2003), si estos aumentan y persisten pueden llegar a transformarse en conductas desadaptativas para el entorno que les rodea tanto para el niño como, en su caso, para el adulto (Van Ameringen, Mancini, y Farvolden, 2003).

En la actualidad, el estudiantado acarrea con situaciones estresantes y de presión, tales como adaptarse a nuevo profesorado y compañero/as, valoraciones rigurosas, acoplarse a la diversidad del centro y a la competitividad entre sus iguales. Todo ello conlleva a desarrollar ideas en torno a la escuela como uno de los entornos de mayor ansiedad y estrés para considerable parte de la población infanto-juvenil (Burnham, 2011; Sung, Puskar, y Sereika, 2006).

Del mismo modo, y en el hilo de lo argumentado, cabe destacar que la tendencia de una conducta ansiosa o a preocuparse en exceso, puede afectar a aspectos de la vida cotidiana del sujeto, tales como a la eficacia en la resolución de problemas, en el desarrollo de la inteligencia interpersonal, en la competencia para relajarse, y por tanto en la calidad de vida y el rendimiento académico.

De hecho, las consecuencias que esto puede acarrear pueden ser de diferente tipo, no solo cognitivo o conductual, tales como preocupaciones o conductas negativistas, sino también es posible percibir la presencia de miedo y ansiedad que afecta a nivel fisiológico, como por ejemplo sudoración, dolores de cabeza, incremento de latidos 
cardíacos, temblores, pérdida del apetito, y trastorno del sueño, entre otros (García-Fernández, Inglés, Martínez-Monteagudo, y Redondo, 2008). Por ello, el constructo ansiógeno en el contexto escolar ha sido de consideración para el profesorado, quienes han podido observar que el alumnado con altos niveles de ansiedad suele ejecutar peor las tareas académicas (Gaudry y Spielberger, 1971).

De ahí, que también se haya puesto énfasis en el estudio de roles mediadores sobre esta variable desadaptativa, contemplando como conclusión que los progenitores, el profesorado y los compañero/as desempeñan una relevante función mediadora sobre la posibilidad que se exhiban estas propensiones ansiosas en el contexto escolar (Cox, 1996), lo que puede desencadenar un desfavorable desarrollo académico del alumnado que las padezcan.

Es importante, conocer cuál es el estado de la cuestión en población ecuatoriana, teniendo en cuenta su déficit actual en su conocimiento.

En el presente estudio existen estudios que examinan las diferencias de ansiedad escolar en función del sexo y curso académico en diversidad de países (Boyd, Ginsburg, Lambert, Colley, y Campbell, 2003; Burnham y Gullone, 1997; Delgado et al., 2019; García-Fernández et al., 2008; Leikanger, Ingul, y Larsson, 2012). No obstante, se carece de estudios previos que examinen estas diferencias en el país de Ecuador.

El objetivo del estudio actual trata de analizar las diferencias de ansiedad escolar en función del sexo y curso en adolescentes ecuatorianos con edades comprendidas entre 15 y 18 años.

En base a la literatura previa:

Hipótesis 1: Se espera que el sexo femenino presente puntuaciones en ansiedad escolar significativamente más altas que el sexo masculino.

Hipótesis 2: Se espera que los estudiantes de último curso presenten puntuaciones en ansiedad escolar más bajas que sus compañeros de cursos inferiores.

\section{MÉTODO}

\section{Participantes}

La muestra fue compuesta bajo un muestreo aleatorio por conglomerados. La muestra total estuvo formada por 720 estudiantes ecuatorianos, con edades comprendidas entre 15 y 18 que cursaban desde $1^{\circ}$ hasta $3^{\circ}$ de Bachillerato, de los cuales $66.9 \%$ son de sexo masculino (véase tabla 1).

La prueba Chi-cuadrado de homogeneidad de la distribución de frecuencias reveló la ausencia de diferencias estadísticamente significativas entre los seis grupos de Sexo x Curso, $\chi 2=2.81, p=.24$. 
Tabla 1. Número de participantes de la muestra total en función del sexo y el curso

\begin{tabular}{lcccc}
\hline & $1^{\circ} \mathrm{BACH}$ & $2^{\circ} \mathrm{BACH}$ & $3^{\circ} \mathrm{BACH}$ & Total \\
& $\mathrm{N}(\%)$ & $\mathrm{N}(\%)$ & $\mathrm{N}(\%)$ & $\mathrm{N}(\%)$ \\
\hline Varones & $150(20.8 \%)$ & $144(20.0 \%)$ & $188(26.1 \%)$ & $482(66.9 \%)$ \\
\hline Mujeres & $60(8.3 \%)$ & $80(11.1 \%)$ & $98(13.6 \%)$ & $238(33.1 \%)$ \\
\hline Total & $210(29.2 \%)$ & $224(31.1 \%)$ & $286(39.7 \%)$ & $720(100.0 \%)$ \\
\hline
\end{tabular}

\section{Instrumentos}

Inventario de Ansiedad Escolar (IAES; García-Fernández et al., 2011): Se trata de un instrumento de medida que trata de evaluar el nivel de ansiedad en población adolescente, concretamente entre los 12 y 18 años de edad, mediante una escala de 5 puntos $(0=$ nunca, $4=$ siempre $)$. El IAES se compone de una tabla de doble entrada.

El eje horizontal es compuesto por 25 ítems referentes a diversas situaciones que se dan en la escuela, los cuales lo conforman 4 dimensiones: FI: Ansiedad ante el fracaso y castigo escolar; FII: Ansiedad ante la evaluación social; FIII: Ansiedad ante la agresión; y FIV: Ansiedad ante la evaluación escolar. El eje vertical lo componen 19 ítems de referentes a la Ansiedad cognitiva (9 ítems), Ansiedad conductual (5 ítems) y Ansiedad psicofisiológica (5 ítems).

Los coeficientes de consistencia interna fueron aceptables para todos los factores de la escala $(\alpha=$ entre .88 y .93), así como para los tres tipos de ansiedad cognitiva $(\alpha=.86)$, conductual $(\alpha=.82)$ y psicofisiológica $(\alpha=.86)$, siendo además su fiabilidad test-retest, en un intervalo de dos semanas, también aceptable $(\alpha=$ entre .78 y .84 para FI, FII, FIII y FIV; y $\alpha=$ entre .74 y .77 para los tres tipos de ansiedad).

\section{Procedimiento}

Se llevó a cabo una entrevista con el equipo directivo y los coordinadores de orientación de los centros con la finalidad de solicitar su permiso y colaboración. Seguidamente, se envió una carta a los progenitores de los participantes para que conocieran el objetivo de la investigación. Tras recibir el consentimiento informado, se dio paso a la administración del instrumento, el cual fue explicado previamente. Este procedimiento fue voluntario y anónimo, y contó con la ayuda del profesorado y la presencia de uno de los investigadores.

\section{Análisis de datos}

Los análisis de datos realizados para observar la significatividad de las diferencias de medias tipificadas, fue realizado mediante el índice d, bajo los criterios propuestos por Cohen (1988), el cual estima que un tamaño del efecto pequeño se da entre .20 y .49 , un tamaño moderado entre .50 y .79 y un tamaño del efecto grande se da en puntuaciones más elevadas que .80 . 


\section{RESULTADOS}

La tabla 2 nos muestra cómo el sexo femenino $(M=283.50)$ presenta un nivel significativamente más elevado de ansiedad escolar que el sexo masculino $(M=201.46)$. Asimismo, esto se repite en los cuatro factores situacionales escolares, así como en cada una de las respuestas de ansiedad escolar (cognitiva, conductual y psicofisiológica).

Cabe destacar que las magnitudes de las diferencias fueron bajas, oscilando entre $d=.49$ para la Ansiedad ante la evaluación escolar y $d=.70$ para la ansiedad ante la agresión y el total de la escala (véase tabla 2).

Tabla 2. Diferencias en las puntuaciones de las situaciones y respuestas de ansiedad escolar según género

\begin{tabular}{lccccccccc}
\hline & \multicolumn{2}{c}{ Hombres } & \multicolumn{2}{c}{ Mujeres } & \multicolumn{2}{c}{ Muestra total } & \multicolumn{3}{c}{$\begin{array}{c}\text { Significación estadística y } \\
\text { magnitud de las diferencias }\end{array}$} \\
\hline & $\mathrm{M}$ & $\mathrm{DE}$ & $\mathrm{M}$ & $\mathrm{DE}$ & $\mathrm{M}$ & $\mathrm{DE}$ & $\mathrm{F}(1,718)$ & $\mathrm{p}$ & $\mathrm{d}$ \\
\hline FI & 26.21 & 24.94 & 47.05 & 35.47 & 33.10 & 30.45 & 84.086 & $<.001$ & .70 \\
\hline FII & 94.05 & 44.64 & 121.18 & 44.37 & 103.02 & 46.31 & 68.550 & $<.001$ & .61 \\
\hline FIII & 48.32 & 35.26 & 71.45 & 42.92 & 55.97 & 39.46 & 61.378 & $<.001$ & .60 \\
\hline FIV & 32.87 & 21.38 & 43.81 & 23.30 & 36.49 & 22.57 & 46.880 & $<.001$ & .49 \\
\hline Cogn & 41.42 & 22.50 & 57.36 & 25.87 & 46.69 & 24.81 & 75.848 & $<.001$ & .67 \\
\hline Cond & 17.93 & 11.89 & 26.02 & 14.49 & 20.61 & 13.35 & 66.799 & $<.001$ & .62 \\
\hline Pfisi & 28.64 & 25.63 & 45.83 & 30.97 & 34.32 & 28.65 & 67.771 & $<.001$ & .61 \\
\hline Tot & 201.46 & 111.08 & 283.50 & 126.13 & 228.58 & 122.43 & 86.747 & $<.001$ & .70 \\
\hline
\end{tabular}

Nota: FI = Ansiedad ante la agresión; FII = Ansiedad ante la evaluación social; FIII = Ansiedad ante el fracaso escolar y sanciones disciplinarias; FIV = Ansiedad ante la evaluación escolar; Cogn = Ansiedad cognitiva; Cond = Ansiedad conductual; Pfisi = Ansiedad psicofisiológica; Tot = Puntuación total.

La tabla 3 muestras las puntuaciones medias en situaciones y respuestas de ansiedad escolar en función del curso académico entre $1^{\circ}$ y $3^{\circ}$ de Bachillerato. Los resultados hallados afirman que las puntuaciones medias más elevadas se dan el grupo más joven $(M=247.78)$, y las menos elevadas en $3^{\circ}$ de Bachillerato $(M=207.11)$, produciéndose un descenso conforme aumenta la edad de los participantes.

Tabla 3. Diferencias de puntuaciones medias en situaciones y respuestas de ansiedad escolar por curso académico

\begin{tabular}{lccccccccc}
\hline & & 1 & & & 2 & & \multicolumn{3}{c}{ Significación estadística } \\
\hline & $\mathrm{M}$ & $\mathrm{DE}$ & $\mathrm{M}$ & $\mathrm{DE}$ & $\mathrm{M}$ & $\mathrm{DE}$ & $\mathrm{F}(2,717)$ & $\mathrm{p}$ & $\eta 2$ \\
\hline FI & 33.26 & 30.19 & 35.43 & 32.32 & 31.15 & 29.07 & 2.082 & .125 & .01 \\
\hline FII & 112.44 & 46.17 & 108.38 & 48.60 & 91.90 & 42.28 & 19.530 & $<.001$ & .05 \\
\hline FIII & 61.23 & 41.65 & 56.35 & 37.92 & 51.81 & 38.66 & 4.735 & .009 & .02 \\
\hline FIV & 40.83 & 24.59 & 37.83 & 22.21 & 32.24 & 20.57 & 13.774 & $<.001$ & .04 \\
\hline Cogn & 48.99 & 23.90 & 48.11 & 25.34 & 43.90 & 24.87 & 4.942 & .007 & .02 \\
\hline Cond & 22.03 & 13.90 & 21.54 & 12.96 & 18.84 & 13.09 & 5.908 & .003 & .02 \\
\hline Pfisi & 39.24 & 31.11 & 35.49 & 28.32 & 29.81 & 26.36 & 9.462 & $<.001$ & .03 \\
\hline Total & 247.78 & 124.29 & 238.01 & 125.23 & 207.11 & 115.82 & 11.366 & $<.001$ & .03 \\
\hline
\end{tabular}

Nota: FI = Ansiedad ante la agresión; FII = Ansiedad ante la evaluación social; FIII = Ansiedad ante el fracaso escolar y sanciones disciplinarias; FIV = Ansiedad ante la evaluación escolar; $\operatorname{Cogn}=$ Ansiedad cognitiva; Cond $=$ Ansiedad conductual; Pfisi $=$ Ansiedad psicofisiológica; Tot = Puntuación total. 
Asimismo, la significación estadística no se observa en todos los casos. Es en el FII (Ansiedad ante la evaluación social), FIV (Ansiedad ante la evaluación escolar), Ansiedad psicofisiológica y en el total de la escala donde se observa esa significación, siendo en todos los casos las puntuaciones más elevadas para $1^{\circ}$ de bachiller, y menos elevadas para $3^{\circ}$ de bachiller.

En la tabla 4 podemos observar las comparaciones post-hoc. Los hallazgos muestran que existen diferencias de pequeña magnitud entre el grupo de $1^{\circ}$ de Bachillerato y de $3^{\circ}$ de Bachillerato para todos los factores y tipos de ansiedad escolar, así como el total de la escala, excepto para la ansiedad ante la agresión. Asimismo, se observan diferencias de baja magnitud en la ansiedad ante la evaluación social $(p=<.001, d=.36)$, la ansiedad ante la evaluación escolar ( $\mathrm{p}=.012, d=.26)$, y el total de la escala de ansiedad escolar $(p=.008, d=.26)$ entre el grupo de $2^{\circ}$ y $3^{\circ}$ de Bachillerato.

Tabla 4. Índice Cohen para contraste post hoc entre grupos de distinta edad y dimensiones de Ansiedad escolar

\begin{tabular}{|c|c|c|c|c|}
\hline & & $1^{\circ}-2^{\circ}$ & $1^{\circ}-3^{\circ}$ & $2^{\circ}-3^{\circ}$ \\
\hline \multirow{2}{*}{ FII } & $\mathrm{p}$ & n.s. & $<.001$ & $<.001$ \\
\hline & d & - & .47 & .36 \\
\hline \multirow{2}{*}{ FIII } & $\mathrm{p}$ & n.s. & .019 & n.s. \\
\hline & d & - & .24 & - \\
\hline \multirow{2}{*}{ FIV } & $\mathrm{p}$ & n.s. & $<.001$ & .012 \\
\hline & d & - & .38 & .26 \\
\hline \multirow{2}{*}{ Cogn } & $\mathrm{p}$ & n.s. & .044 & n.s. \\
\hline & d & - & .24 & - \\
\hline \multirow{2}{*}{ Cond } & $\mathrm{p}$ & n.s. & .018 & n.s. \\
\hline & d & - & .24 & - \\
\hline \multirow{2}{*}{ Pfisi } & $\mathrm{p}$ & n.s. & $<.001$ & n.s. \\
\hline & d & - & .28 & - \\
\hline \multirow[b]{2}{*}{ Tot } & $\mathrm{p}$ & n.s. & $<.001$ & .008 \\
\hline & $\frac{P}{d}$ & - & .34 & .26 \\
\hline
\end{tabular}

Nota: FII = Ansiedad ante la evaluación social; FIII = Ansiedad ante el fracaso escolar y sanciones disciplinarias; FIV = Ansiedad ante la evaluación escolar; $\operatorname{Cogn}=$ Ansiedad cognitiva; Cond $=$ Ansiedad conductual; Pfisi $=$ Ansiedad psicofisiológica; Tot $=$ Puntuación total .

\section{CONCLUSIONES/DISCUSIÓN}

El objetivo del presente trabajo era observar si existen diferencias estadísticamente significativas en las puntuaciones medias tanto en situaciones como en respuestas de ansiedad escolar en función del sexo y el curso en el que se encuentra matriculado el grupo de alumnado participante.

En este sentido, y con respecto a las diferencias del constructo estudiado en función del sexo, los resultados muestran que son las chicas las que presentan un nivel significativamente mayor de ansiedad escolar con respecto al sexo masculino. Del mismo modo, los hallazgos obtenidos mostraron puntuaciones significativamente más altas en el sexo femenino con respecto al sexo masculino tanto en cada una de las dimensiones situacionales que se pueden dar en la escuela, como en cada una de los tipos de ansiedad escolar que evalúa el IAES (García-Fernández et al., 2011). 
De este modo, se confirma la primera de las hipótesis marcadas en el trabajo, tal y como afirman diversos trabajos anteriores que miden tanto la ansiedad escolar como la ansiedad generalizada en países diversos en población adolescente afroamericana (Boyd et al., 2003), estadounidense (Burnham y Gullone, 1997), francesa (Delgado et al., 2019), austriaca (Freudenthaler et al., 2008), española (García-Fernández et al., 2011), y noruega (Leikanger et al., 2012). En este sentido, Avero et al. (2000) afirman que esta situación puede deberse a cuestiones culturales tales como la mayor capacidad de expresión de sentimientos y emociones de las chicas frente al aliento hacia el sexo masculino a mostrar sentimientos de valentía, competencia y agresividad.

Por otra parte, y referente a las diferencias de edad, se observa que el grupo matriculado en primero de Bachillerato presenta puntuaciones más altas de ansiedad ante la evaluación social y la evaluación escolar, la ansiedad psicofisiológica y el total de la escala de ansiedad escolar, mientras que desciende en los estudiantes de segundo y tercer año de Bachillerato.

De este modo, se cumple la segunda de las hipótesis planteadas. A este respecto, Steinhausen et al. (2008) demostraron en adolescentes suizos que el miedo y la fobia a la escuela disminuyó en el alumnado con el tiempo. Por lo que, estos resultados son concordantes con los del presente estudio donde la ansiedad escolar disminuye conforme se aumenta la edad.

No obstante, se debe tener en cuenta la importancia de este estudio en cuanto a la repercusión que ello puede tener en el alumnado, como se ha comprobado con estudios previos anteriormente mencionados (Batxer et al., 2013; Burnham, 2009; Beesdo et al., 2009; Dadds et al., 1999; García-Fernández et al., 2008; Gaudry y Spielberg, 1971; Steel et al., 2014; Sung et al., 2006; Van Ameringen et al., 2003).

De hecho, la literatura previa afirma que cuando esta ansiedad se da en población adulta y adolescente, su desarrollo es de carácter más gradual, pero además se presenta con mayor intensidad, por lo que su pronóstico es más grave (Echeburúa- Odriozola, 2009; García-Fernández et al., 2008). De ahí, la necesidad de conocer estos resultados, así como los tratamientos oportunos. En este sentido, además de orientaciones de apoyo educativo (Last et al., 1998) son las terapias cognitivoconductuales las que producen mayor eficacia (Heyne et al., 2004; King et al., 2000).

Asimismo, se observa que excepto para la ansiedad ante la agresión, se identifican diferencias significativas en todos los tipos de ansiedad y todos los factores de la ansiedad escolar entre los grupos de $1^{\circ}$ y $3^{\circ}$ de bachillerato, siendo además significativas estas diferencias entre $2^{\circ}$ y $3^{\circ}$ para la ansiedad ante la evaluación social y la evaluación escolar, así como el total de la escala de ansiedad escolar.

Estos datos indican, por una parte, rasgos culturales y sugieren el inicio de una investigación basada en el estudio sobre variables de la frecuencia de episodios de violencia en las instituciones escolares en función de la alta y baja ansiedad escolar en 
participantes de Ecuador, para determinar si el no observar diferencias entre edades se debe a un exceso de episodios violentos al que están mayormente acostumbrados, o por el contrario a una carencia debido a que estos episodios son menos frecuentes.

Asimismo, cabe destacar que el hecho de observarse en población adolescente que, a menor edad, mayor ansiedad ante la evaluación social, puede deberse a varios aspectos. Por una parte, Rao et al. (2007) afirman que es la etapa de la adolescencia en la que se desarrolla una mayor capacidad empática. Por otro lado, se establecen esas expectativas de adaptación social debido a que se enmarcan en una nueva etapa académica, donde también existe esa necesidad de establecer nuevos vínculos entre iguales (Inglés et al., 2010). Del mismo modo, la presencia de mayores demandas académicas, aumentaría con ello el miedo de la evaluación escolar y el rendimiento académico (Inglés et al., 2010; Rao et al., 2007), corroborando los hallazgos obtenidos en el estudio.

Cabe destacar que, pese a la importancia de los resultados obtenidos, el trabajo tiene varias limitaciones que deben tenerse en cuenta para futuras investigaciones en el mismo ámbito de estudio. Por una parte, sería conveniente para futuros estudios contemplar las posibles patologías y los aspectos culturales de los participantes.

Por otra parte, además de medir el constructo con instrumentos como el empleado en el presente trabajo, herramienta válida y fiable (García-Fernández et al., 2011), deberían emplearse en futuros estudios técnicas de observación y entrevistas cualitativas con los participantes, obteniendo así una perspectiva más amplia que dé lugar a conocer de modo más evidente cuál es el grado de intensidad de la ansiedad escolar en cada uno de los casos estudiados.

Sin embargo, y pese a observar dichas limitaciones, los resultados observados ponen de manifiesto la necesidad de evaluar, estos niveles de ansiedad escolar mayormente en las chicas ecuatorianas, así como en grupos adolescentes de alrededor de 15 años de edad, con objeto de observar si se repiten los hallazgos contemplados, así como de poner en práctica programas que reduzcan estos niveles de ansiedad escolar y sus posibles riesgos comórbidos (Batxer et al., 2013; García-Fernández et al., 2008; Gaudry y Spielberg, 1971; Steel et al., 2014).

De este modo, los hallazgos del presente estudio, contribuyen a un nuevo conocimiento científico en el país de Ecuador, permitiendo seguir con su estudio y diseñar programas más específicos para la prevención y disminución de la ansiedad escolar en adolescentes. 


\section{REFERENCIAS}

Avero, P., Fernández-Valdés, A., López-Curbelo, M., Bethencourt, J.M., y Matud, P. (2000). Reactividad emocional en una fobia específica: diferencias y patrones de concordancia en el triple sistema de respuestas en función del género. Ansiedad y Estrés, 6(1), 93-104.

Batxer, A.J., Scott, K.M., Vos, T., y Whiteford, H.A. (2013). Global prevalence of anxiety disorders: a systematic review and meta-regression. Psychological Medicine, 43(5), 897-910. doi: $10.1017 / \mathrm{S} 003329171200147 \mathrm{X}$

Beesdo, K., Knappe, S., y Pine, D.S. (2009). Anxiety and anxiety disorders in children and adolescents: developmental issues and implications for DSM-V. The Psychiatric Clinics of North America, 32(3), 483-524. doi: 10.1016/j.psc.2009.06.002

Boyd, R.C., Ginsburg, G.S., Lambert, S.F., Cooley, M.R., y Campbell, K.D.M. (2003). Screen for Child Anxiety Related Emotional Disorders (SCARED): Psychometric properties in an african-american parochial high school sample. Journal of the American Academy of Child y Adolescent Psychiatry, 42(10), 1188-1196. doi: 10.1097/00004583-20031000000009

Burnham, J.J. (2011). Contemporary fears of children and adolescents: coping and resiliency in the 21 st century. Journal of Counseling and Development, 87(1), 28-35. doi. 10.1002/j.15566678.2009.tb00546.x

Burnham, J.J., y Gullone, E. (1997). The Fear Survey Schedule for Children-II: a psychometric investigation with american data. Behavior Research and Therapy, 35(2), 165-173. doi: 10.1016/S0005-7967(96)00089-7

Cohen, J. (1988). Statistical power analysis for the behavioral sciences. Second Edition Hillsdale, NJ: Lawrence Erlbaum Associates, Publishers.

Costello, E.J., Egger, H.L., y Angold, A. (2005). The developmental epideomiology of anxiety disorders: phenomenology, prevalence, and comorbidity. Child and Adolescent Psychiatric clinics of North America, 14(4), 631-648. doi: 10.1016/j.chc.2005.06.003

Cox, B.J. (1996). The nature and assessment of catastrophic thoughts in panic disorder. Behavior Research and Therapy, 34(4), 363-374.

Dadds, M.R., Holland, D.E., Laurens, K.R., Mullins, M., Barrett, P.M., y Spence, S.H. (1999). Early intervention and prevention of anxiety disorders in children: results at 2-year follow-up. Journal of Consulting and Clinical Psychology, 67(1), 145-150.

Delgado, B., García-Fernández, J.M., Martínez-Monteagudo, M.C., Inglés, C.J., Marzo, J.C., La Greca, A.M., y Hugon, M. (2019). Social Anxiety Scale for Adolescents and School Anxiety Inventory: pscyhometric properties in french adolescents. Child Psychiatry and Human Development, 50(1), 13-26. doi: 10.1007/s10578-018-0818-4

Echeburúa-Odriozola, E. (2009). Trastornos de ansiedad en la infancia. Madrid: Pirámide.

Freudenthaler, H.H., Spinath, B., y Neubauer, A.C. (2008). Predicting school achievement in boys and girls. European Journal of Personality, 22(3), 231-245. doi: 10.1002/per.678

García-Fernández, J.M., Inglés, C.J., Martínez-Monteagudo, M.C., Marzo, J.C., y Estévez, E. (2011). Inventario de Ansiedad Escolar: validación en una muestra de estudiantes de Educación Secundaria. Psicothema, 23(2), 301-307.

García-Fernández, J.M., Inglés, C.J., Martínez-Monteagudo, M.C., y Redondo, J. (2008). Evaluación y tratamiento de la ansiedad escolar en la infancia y adolescencia. Psicología Conductual, 16(3), 413-437.

García-Fernández, J.M., Martínez-Monteagudo, M.C., e Inglés, C. (2011). Diferencias según sexo y curso en ansiedad escolar: estudio con una muestra de estudiantes españoles de educación secundaria. Ansiedad y Estrés, 17(2/3), 137-148.

Gaudry, E., y Spielberg, C.D. (1971). Anxiety and educational achievement. New York: Wiley. 
Heyne, D., King, N.J., y Tonge, B. (2004). School refusal. En T.H. Ollendick y J.S. March (dirs.), Phobic and anxiety disorders in children and adolescents: a clinician's guide to effective psychosocial and pharmacological interventions (pp. 236-271). Nueva York: Oxford University Press.

Inglés, C.J., Piqueras, J.A., García-Fernández, J.M., García-López, L.J., Delgado, B., y Ruiz- Esteban, C. (2010). Diferencias de género y edad en respuestas cognitivas, psicofisiológicas y motoras de ansiedad social en la adolescencia. Psicothema, 22(3), 376381.

Jiménez-Ayala, C.E. (2017). Ansiedad escolar y su relación con las atribuciones académicas, el autoconcepto, perfeccionismo y agresividad en una muestra de estudiantes ecuatorianos de 12 a 18 años (Tesis doctoral). Universidad de Alicante, España.

Kessler, R.C., Angermeyer, M., Anthony, J.C., De Graaf, R., Demyttenaere, K., Gasquet, I., ... Bedirhan-Üstün, T. (2007). Lifetime prevalence and age-of-onset distributions of mental disorders in the World Health Organization's World Mental Health Survey Initiative. World Psychiatry, 6(3), 168-176.

King, N.J., Tonge, B.J., Heyne, D., y Ollendick, T.H. (2000). Research on the cognitivebehavioral treatment of school refusal: A review and recommendations. Clinical Psychology Review, 20, 495-507.

Last, C.G., Hansen, C., y Franco, N. (1998). Cognitive behavioural treatment of school phobia. Journal of the American Academy of Child and Adolescent Psychiatry, 37, 404-411.

Leikanger, E., Ingul, J.M., y Larsson, B. (2012). Sex and age-related anxiety in a community sample of Norwegian adolescents. Scandinavian Journal of Psychology, 53(2), 150-157. doi; 10.111/j.1467-9450.2011.00915.x

Méndez, F.X., Inglés, C.J., Hidalgo, M.D., García-Fernández, J.M., y Quiles, M.J. (2003). Los miedos en la infancia y la adolescencia: Un estudio descriptivo. Revista Electrónica de Motivación y Emoción, 6(13).

Ollendick, T.H., y Seligman, L.D. (2005). Anxiety disorders. En C. Gillberg, R. Harrington, y H.C. Steinhausen (Eds.), A clinician's handbook of child and adolescent psychiatry (pp. 144-187). Cambridge: Cambridge University Press.

Rao, P.A., Beidel, D.C., Turner, S.M., Ammerman, T., Crosby, L.E., y Sabellee, F.R. (2007). Social anxiety disorder in childhood and adolescence: descriptive psychopathology. Behavior Research and Therapy, 45, 1181-1191.

Steel, Z., Marnane, C., Iranpour, C., Chey, T., Jackson, J.W., Patel, V., y Silove, D. (2014). The global prevalence of common mental disorders: a systematic review and meta-analysis 1980-2013. International Journal of Epidemiology, 43(2), 476-493. doi: 10.1093/ije/dyu038

Steinhausen, H.C., Müller, N., y Metzcke, C.W. (2008). Frequency, stability and differentiation of self-reported school fear and truancy in a community sample. Child and Adolescent Psychiatry and Mental Health, 2(1), 1-11. doi: 10.1186/1753-2000-2-17

Sung, K.M., Puskar, K.R., y Sereika, S. (2006). Psychosocial factors and coping strategies of adolescents in a rural Pennsylvania high school. Public Health Nursing, 23(6), 523-530. doi 10.1111/j.1525-1446.2006.00589.x

Van Ameringen, M., Mancini, C., y Farvolden, P. (2003). The impact of anxiety disorders on educational achievement. Journal of Anxiety Disorders, 17(5), 561-571.

Recibido: 25 de noviembre de 2019

Recepción Modificaciones: 20 de diciembre de 2019

Aceptado: 12 de enero de 2020 